\title{
Simulation of anyonic fractional statistics of Kitaev's toric model in circuit QED
}

\author{
ZHENG-YUAN XUE \\ Laboratory of Quantum Information Technology, ICMP and School of Physics and Telecommunication Engineering, \\ South China Normal University, Guangzhou 510006, China
}

PACS 03.67.Lx - Quantum computation architectures and implementations

PACS 42.50.Dv - Quantum state engineering and measurements

PACS 85.25.Cp - Josephson devices

\begin{abstract}
Since the anyonic excitations in the Kitaev toric model are perfectly localized quasiparticles, it is possible to generate dynamically the ground state and the excitations of the model Hamiltonian to simulate the anyonic interferometry. We propose a scheme in circuit QED to simulate the interferometry. The qubit-cavity interaction can be engineered to realize effective state control as well as the controlled dynamics of qubits, which are sufficient to prepare the ground states, create and remove the anyonic excitation, and simulate the anyonic interferometry. The simplicity and high fidelity of the operations used open the very promising possibility of simulating fractional statistics of anyons in a macroscopic material in the near future.
\end{abstract}

Introduction. - Anyons are exotic quasiparticles living in two dimensions with fractional statistics [1]. A paradigmatic system for the existence of anyons is a kind of so-called fractional quantum Hall states [2]. Alternatively, artificial spin lattice models are also promising for observing these exotic excitations [3 5]. Recently, with the potential applications in topological quantum com'putation, anyons have attracted strong renewed interests [6] 15. However, a direct observation of fractional statistics associated with anyon braiding is hard. For the Kitaev toric model, it has been proposed 11 an alternative, i.e., to generate dynamically the ground state and the excitations of the model Hamiltonian, instead of direct ground-state cooling, to simulate the anyonic interferometry. The anyons are perfectly localized quasiparticles in that model Hamiltonian, therefore one do not need a large system for implementing their braiding operations. Indeed, small-scale system for proof-of-principle simulation of the anyonic fractional statistics was realized in optical systems [12 14. This kind of experiment represents an important step toward the long pursued goal to simulate fractional statistics of quasiparticles in a macroscopic material, which is critical for future implementation of topological quantum computer, providing the ability to scale up to large systems.

Superconducting circuit is one of the most promising candidates serving as hardware implementation of quan- tum computers [16]. In this paper, we propose a scheme in circuit QED to simulate the interferometry. The qubitcavity interaction can be engineered to realize effective state control as well as the controlled dynamics of the two-level systems, which are sufficient [15] to prepare the ground states of the toric Hamiltonian, create and remove the anyonic excitation, and simulate the anyonic interferometry. The simplicity and high fidelity of the operations used may open the very promising possibility of simulating fractional statistics of anyons in a macroscopic superconducting phase qubit in the near future.

Quantum dynamics in circuit QED. - The superconducting charge qubit considered here consists of a small superconducting box with excess Cooper-pair charges, formed by a symmetric SQUID with the capacitance $C_{J}$ and Josephson coupling energy $E_{J}$, pierced by an external magnetic flux $\Phi$. A control gate voltage $V_{g}$ is connected to the system via a gate capacitor $C_{g}$. Focus on the charge regime, at temperatures much lower than the charging energy $\left[E_{c}=2 e^{2} /\left(C_{g}+2 C_{J}\right)\right]$ and restricting the induced charge $\left[\bar{n}=C_{g} V_{g} /(2 e)\right]$ to the range of $\bar{n} \in[0,1]$, only a pair of adjacent charge states on the island are relevant. The qubits are capacitively coupled to a transmission line resonator which forms a 1D cavity. For simplicity, we here assume that the cavity has only a single mode that plays a role. To obtain maximum coupling strength, they are fab- 
ricated close to the voltage antinodes of the cavity. This coupling is determined by the gate voltage, which contains both the dc contribution and a quantum part. The qubits are working at their optimal points, where they are immune to the charge noise and possess long decoherence time. In the qubit eigenbasis, neglecting fast oscillating terms using the rotating-wave approximation, the Hamiltonian describes this scenario now takes the usual JaynesCummings form 17.

$$
H_{J C}=\omega_{r} a^{\dagger} a+\frac{\nu}{2} \sigma^{z}-g\left(a^{\dagger} \sigma^{-}+a \sigma^{+}\right),
$$

where we have assume $\hbar=1, \nu$ is the qubit energy splitting, $g$ is the coupling strength of a qubit to the cavity, $\omega_{r}, a$ and $a^{\dagger}$ is the frequency, annihilation and creation operator of the cavity field, respectively. Note that the qubit frequency can be tune within a large range by the external magnetic field. Therefore, selected qubit-cavity interaction can be achieved.

The evolution operator of qubit-cavity interaction Hamiltonian in Eq. (11) is given by

$$
U(t)=\left(\begin{array}{cccc}
1 & 0 & 0 & 0 \\
0 & \cos \theta & -i \sin \theta & 0 \\
0 & -i \sin \theta & \cos \theta & 0 \\
0 & 0 & 0 & 1
\end{array}\right)
$$

where $\theta=g t$, and it results in an oscillation between the qubit and cavity states.

Meanwhile, driving in the form of

$$
h=\varepsilon(t) a^{\dagger} e^{-i \omega_{d} t}+\varepsilon^{*}(t) a e^{i \omega_{d} t}
$$

on the resonator can be obtained [17 by capacitively coupling it to a microwave source with frequency $\omega_{d}$ and amplitude $\varepsilon(t)$. Depending on the frequency, phase, and amplitude of the drive, different logical operations for qubit can be realized.

To get fast gate, we work with large amplitude driving fields, where quantum fluctuations are very small compare with the drive amplitude, and thus the drive can be considered as a classical field. In this case, it is convenient to displace the field operators using the time-dependent displacement operator [18]:

$$
D(\alpha)=\exp \left(\alpha a^{\dagger}-\alpha^{*} a\right) .
$$

Choosing $i \dot{\alpha}=\omega_{r} \alpha+\varepsilon(t) e^{-i \omega_{d} t}$ to eliminate the direct drive on the resonator, by $h$, from the effective Hamiltonian, then the displaced Hamiltonian for a qubit reads 17.

$$
H_{D}=\omega_{r} a^{\dagger} a+\frac{\nu}{2} \sigma^{z}-g\left[(a+\alpha) \sigma^{+}+H . c .\right] .
$$

When the drive amplitude is independent of time, and change to a frame rotating at $\omega_{d}$, the displaced Hamiltonian reads

$$
H_{\mathrm{RF}}=\delta a^{\dagger} a+\frac{\Delta}{2} \sigma_{z}+\frac{\Omega}{2} \sigma_{x}-g\left(a \sigma^{+}+a^{\dagger} \sigma^{-}\right) .
$$

where $\delta=\omega_{r}-\omega_{d}, \Delta=\nu-\omega_{d r}$, and $\Omega=2 g \varepsilon / \delta$ is the Rabi frequency. In the dispersive regime $\delta \gg g$, the effective Hamiltonian is [17]

$$
\hat{H}_{x}=\delta \hat{a}^{\dagger} \hat{a}+\frac{\Delta+g^{2} / \delta}{2} \hat{\sigma}_{z}+\frac{\Omega}{2} \hat{\sigma}_{x} .
$$

By choosing $\Delta+g^{2} / \delta=0$, the Hamiltonian (6) evolves as a rotation around the $x$ axis. The gate speed scales as $t_{x} \sim 1 / \Omega$, which have already been experimentally verified [19.

We now turn to consider that the drive is sufficiently detuned from the qubit $|\Delta| \gg \Omega$. Then, the effective Hamiltonian from Eq. (5) is [17.

$$
\hat{H}_{z}=\delta \hat{a}^{\dagger} \hat{a}+\frac{\chi}{2} \hat{\sigma}_{z},
$$

which generates rotations around $z$ axis at a rate $\chi=$ $\Delta+g^{2} / \delta+\Omega^{2} /(2 \Delta)$, and the gate speed scales as $t_{z} \sim 1 / \chi$.

From the above analysis, we can see that switch on and off the qubit-cavity coupling can be achieved by tuning the external driven field. In this way, we can obtain individual manipulation of qubits. It worth noticing that the dispersive regime in single-qubit operation is induced by the detuning with respect to the driving, which will not slow the gate speed as in the dispersive coupling of qubits via virtual excitation of the cavity mode [17. Specifically, $t_{x} \sim 1 / g$ when $\epsilon \sim \delta / 2$.

Note that the iSWAP gate between the cavity and $j$ th qubit can be obtained from evolution operator (2) when $\tau=\pi /(2 g)$. Together with single-qubit rotations, it can be used to generate cluster states more efficiently than that of controlled phase flip gate [20,21]. The operation used for the cavity and qubit system is

$$
\begin{aligned}
U^{c} & =I \otimes Z_{(\pi / 2)} \times U(\tau) \times I \otimes Z_{(\pi / 2)} \\
& =\left(\begin{array}{cccc}
1 & 0 & 0 & 0 \\
0 & 0 & 1 & 0 \\
0 & 1 & 0 & 0 \\
0 & 0 & 0 & -1
\end{array}\right)
\end{aligned}
$$

where $Z_{(\pi / 2)}$ is a $\pi / 2$ rotation around the $z$ axis of the qubit state. This gate makes the two involved parties linked by a controlled phase flip gate and at the same time it swaps their states. It is worth noticing that both singlequbit and iSWAP operation times are several $n$ s which is much smaller compare to typical decoherence time of the qubit 22] and cavity 23], both of them being on the order of $\mu \mathrm{s}$.

Ground states preparation. - In the following, we show how to prepare the ground states of the toric model [4] with this gate. The toric code is defined as the ground level of a stabilizer Hamiltonian

$$
H=-\sum_{v} A_{v}-\sum_{p} B_{p}
$$



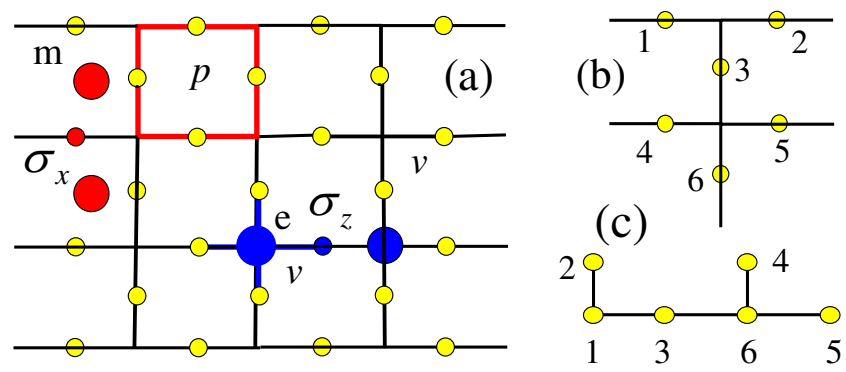

Fig. 1: (Color online) The toric model. (a). It is defined as the ground state of a stabilizer Hamiltonian on a square lattice with spins at the edges of a square lattice. The commuting vertices (plaquettes), as indicated by a blue cross (red rectangle), are the stabilizers. A single $\sigma_{x}\left(\sigma_{z}\right)$ gate on a spin, indicated by a small red (blue) circle, can create a pair of magnetic (electric) defects excitation, indicated by a big red (blue) circle. (b). The smallest system for implementation of the anyon braiding operation, the ground state of which is equivalent under local single-bit operations to a graph state in (c).

on a square lattice with spins, realized as superconducting qubits here, at the edges of a square lattice as shown in Fig. (12). The sum is over the mutually commuting stabilizers

$$
A_{v}=\prod_{i \in v} \sigma_{i}^{x}, \quad B_{p}=\prod_{j \in p} \sigma_{j}^{z}
$$

where $v$ runs over all vertices and $p$ over plaquettes, as indicated by a blue cross and a red rectangle in Fig. (1h). The ground state $|\varphi\rangle_{g}$ is characterized by $A_{v}=B_{p}=1$. Plaquette and vertex excitations $|\varphi\rangle_{e, m}$, also called magnetic and electric defects and indicated in Fig. (17) by bigger red and blue fulled circles, are characterised by $B_{p}=-1, A_{v}=-1$ and they are mutual Abelian anyons. Therefore, apply single $\sigma_{x}$ or $\sigma_{z}$ can create these quasiparticle excitations. These excitations always appear in pairs, at the ends of strings of $\sigma_{x}$ and $\sigma_{z}$ operators applied on a ground state. To apply $\sigma_{x}$ or $\sigma_{z}$ to a spin, we need to turn on the qubit-cavity interaction in Eq. (6) or (7).

For the toric model, the excitations are perfectly localized. Therefore, for a proof-of-principle simulation of anyons and their braiding statistics, the minimum implementation only needs six spins [11, as the graph shown in Fig. 1(b). The ground state of which is equivalent under local single-bit operations to a graph state in Fig. 1(c). We next show how to prepare the ground state. We work with a appropriate 3 -body vertex and plaquette operators along the boundary providing for a two-dimensional code space. We start with a well defined state, i.e., the cavity and spins $1,2,3,5$, and 6 are initialized to the state of $|+\rangle=(|0\rangle+|1\rangle) / \sqrt{2}$ while spin 4 is prepared in the ground state $|g\rangle$. The process is illustrated in Fig. (2). We first sequentially apply $U^{c}$ on cavity and spins $2,1,3,6$, and 5 , which results in the five qubit cluster state and leave the cavity to the $|0\rangle$ state. As illustrated in the Fig. (22), after each $U^{c}$, the cavity is at the right end of the cluster.

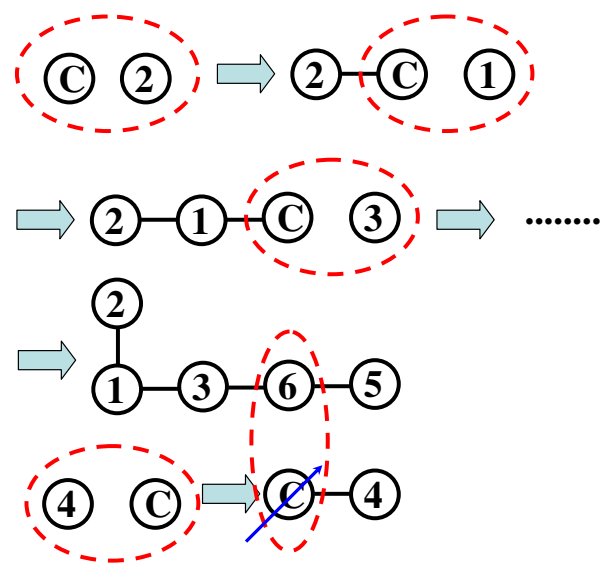

Fig. 2: (Color online) The process of generating the ground state graph with six spins. Each dashed circle denotes a $U^{c}$ operation and each bond means the two are linked by a controlled phase flip gate. Circle with capital letter "C" and numbers denote the cavity and spins, respectively. The blue arrow across the circle represents "X" measurement on the cavity.

Then, apply $U^{c}$ on spin 4 to create an bipartite entanglement. At last apply $U^{c}$ on spin 6 to fuse the two entangled states followed by an $X$ measurement on the cavity state. After these steps, the six spins are now prepared in the graph state as in Fig. (15). Measurement on the cavity can be implemented by swapping the state of the cavity to another spin and then measure the spin state.

Controlled dynamics and anyon interference. This operation in Eq. (8) can also be used to realize controlled rotation $U_{z}=|0\rangle\left\langle 0\left|\otimes \sigma_{z}+\right| 1\right\rangle\langle 1| \otimes I$ of the qubit about the $z$ axis under the control of the cavity states. It goes as following: 1) Prepare the cavity state to the state of $(|0\rangle+|1\rangle)$. Here, we need to engineer the cavity number states, which cab be achieved by swapping an ancillary qubit states with that of the cavity. 2) Excite the qubit excited state $|e\rangle$ to an ancillary level (other than $|g\rangle$ and $|e\rangle)$. Ancillary levels have already been used in a recent experimental demonstration of gates in circuit QED [22]. 3) Apply $U^{c}$ for the cavity and the target spin for a time of $\pi / S$. Note that $U_{x}=|0\rangle\left\langle 0\left|\otimes \sigma_{x}+\right| 1\right\rangle\langle 1| \otimes I$ is equivalent to $U_{z}$ up to local single-qubit operation on the spin [10]: $U_{x}=H U_{z} H$. It is note that our controlled operations are different from that of Refs. 10,15. The essential of the controlled operations is to obtain state-dependent dynamics, in this sense, the effect of our controlled operations are the same as their's.

With these controlled operators and rotating gates of the qubits, it is sufficient to create and remove the excitations and to simulate the anyonic interferometry of the toric model. To create a superposition of the ground and excite state, we need an ancillary degree of freedom, realized by the cavity here, then conditional excitation $\sim\left(|\varphi\rangle_{g}|1\rangle+\eta|\varphi\rangle_{e, m}|0\rangle\right)$ can be achieved, where $\eta$ is determined by the relative amplitude of the controlled gate. It is obvious that $\eta=1$ is the controlled operation $U_{x, z}$. To 

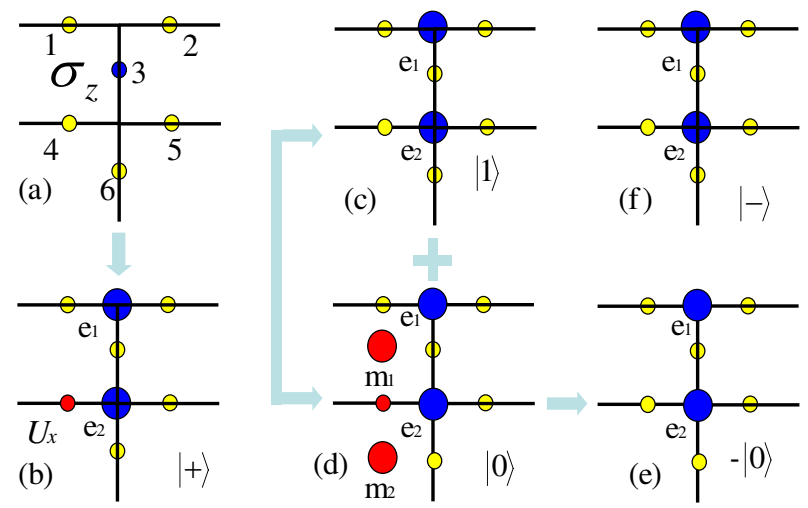

Fig. 3: (Color online) The minimal anyonic interferometry. The label of spins (yellow small circles) is indicated in (a). Applying a $\sigma_{z}$ gate to spin 3 , creates two electric defects $e_{1}$ and $e_{2}$, indicated as big blue circles in (b). With the cavity in state $|+\rangle$, applying a $U_{x}$ gate on spin 4 conditionally create two magnetic defects $m_{1}$ and $m_{2}$, i.e. the system now in the superposition of (c) and (d). With the cavity in state $|+\rangle$, applying $U_{x}$ gates on cavities and spins $6,5,3$ and 4 sequentially result in the superposition of (c) and (e), which is equivalent to $(f)$.

apply $U_{z}$ to a spin, we can get controlled magnetic defects excitation: $U_{x}|\varphi\rangle_{g}|+\rangle \sim\left(|\varphi\rangle_{g}|1\rangle+|\varphi\rangle_{m}|0\rangle\right)$. Similarly, one can also create controlled electric defects excitation by $U_{z}|\varphi\rangle_{g}|+\rangle \sim\left(|\varphi\rangle_{g}|1\rangle+|\varphi\rangle_{e}|0\rangle\right)$.

Once the toric code state is prepared, one can simulate the fractional statistical phase of the anyons through a Ramsey-type interference experiment. The simplest anyonic interferometry [11] is shown in Fig. (3). Apply a $\sigma_{z}$ gate to spin 3 , creates two electric defects $e_{1}$ and $e_{2}$. With the cavity in state $|+\rangle$, apply a $U_{x}$ gate on spin 4 creates the superposition states of (c) and (d). Apply $U_{x}$ gates on cavities and spins $6,5,3$ and 4 sequentially will move $m_{2}$ around $e_{2}$ and finally fusing with $m_{1}$ to vacuum. By this braiding, the -1 factor appears in the cavity state, i.e. the system now in the superposition of (c) and (e), which is equivalent to (f). In this interferometry the cavity state will change to $|-\rangle=H|1\rangle$ from the state of $|+\rangle$. This phase factor on the cavity state is solely due to the mutually fractional statistics of anyons and can be detected unambiguously in experiment. This detection method is similar with that of Ref. [15].

Conclusion. - In summary, we propose a scheme in circuit QED to simulate the fractional statistics of anyons of Kitaev's toric code model. The qubit-cavity interaction can be engineered to realize effective state control as well as the controlled dynamics of qubits, which are sufficient to prepare the ground states, create and remove the anyonic excitation, and simulate the anyonic interferometry. The simplicity and high fidelity of the operations used here open the very promising possibility of simulating fractional statistics of anyons in a macroscopic material in the near future.
$* * *$

I thank Prof. Shi-Liang Zhu for many helpful suggestions. This work was supported by the NSFC (No. 11004065), the NSF of Guangdong Province (No. 10451063101006312), and the Startup Foundation of SCNU (No. S53005).

\section{REFERENCES}

[1] F. Wilczek, Phys. Rev. Lett. 48, 1144 (1982).

[2] C. Nayak, S. H. Simon, A. Stern, M. Freedman, and S. Das Sarma, Rev. Mod. Phys. 80, 1083 (2008).

[3] X.-G. Wen, Phys. Rev. Lett. 90, 016803 (2003); Quantum Field Theory of Many-Body Systems (Oxford University Press, Oxford) 2004.

[4] A. Kitaev, Ann. Phys. (N.Y.) 303, 2 (2003); ibid. 321, 2 (2006).

[5] Z.-Y. Xue, S.-L. Zhu, J. Q. You, and Z. D. Wang, Phys. Rev. A 79, 040303(R) (2009); Y. Yu and Y. Li, J. Phys. A: Math. Theor. 43, 105306 (2010).

[6] L.-M. Duan, E. Demler, and M. D. Lukin, Phys. Rev. Lett. 91, 090402 (2003).

[7] A. Micheli, G. K. Brennen, and P. Zoller, Nature Phys. 2, 341 (2006).

[8] J. Q. You, X.-F. Shi, X. Hu, and F Nori, Phys. Rev. B 81, 014505 (2010).

[9] C. Zhang, V. W. Scarola, S. Tewari, and S. Das Sarma, Proc. Natl. Acad. Sci. U.S.A. 104, 18415 (2007).

[10] L. Jiang, G. K. Brennen, A. V. Gorshkov, K. Hammerer, M. Hafezi, E. Demler, M. D. Lukin, and P. Zoller, Nature Phys. 4, 482 (2008).

[11] Y.-J. Han, R. Raussendorf, and L.-M. Duan, Phys. Rev. Lett. 98, 150404 (2007).

[12] C.-Y. Lu, W.-B. Gao, Otfried Gühne, X.-Q. Zhou, Z.-B. Chen, and J.-W. Pan, Phys. Rev. Lett. 102, 030502 (2009).

[13] J. K. Pachos, W. Wieczorek, C. Schmid, N. Kiesel, R. Pohlner, and H. Weinfurter, New J. Phys. 11, 083010 (2009).

[14] J. Zhang, C. Xie, K. Peng, and P. van Loock, Phys. Rev. A 78, 052121 (2008).

[15] M. Aguado, G. K. Brennen, F. Verstraete, and J. I. Cirac, Phys. Rev. Lett. 101, 260501 (2008).

[16] J. Q. You and F. Nori, Phys. Today 58, (11) 42 (2005).

[17] A. Blais, J. Gambetta, A. Wallraff, D. I. Schuster, S. M. Girvin, M. H. Devoret, and R. J. Schoelkopf, Phys. Rev. A 75, 032329 (2007).

[18] M. O. Scully and M. S. Zubairy, Quantum optics (Cambridge University Press, New York, 1997), chapt. 2.

[19] A. Wallraff et al., Nature (London) 431, 162 (2004).

[20] T. Tanamoto, Y.-X. Liu, X. Hu, and F. Nori, Phys. Rev. Lett. 102, 100501 (2009).

[21] L.-B. Yu, Z.-Y. Xue, Z. D. Wang, Y. Yu, and S.-L. Zhu, arXiv:0904.1275 (2009), to be published in Eur. J. Phys. D, DOI: 10.1140/epjd/e2010-00258-5.

[22] L. DiCarlo et al., Nature (London) 460, 240 (2009).

[23] L. Frunzio, A. Wallraff, D. I. Schuster, J. Majer, and R. J. Schoelkopf, IEEE Trans. Appl. Superconductivity 15, 860 (2005). 